\title{
Resveratrol prevents the impairment of advanced glycosylation end products (AGE) on macrophage lipid homeostasis by suppressing the receptor for AGE via peroxisome proliferator-activated receptor $\gamma$ activation
}

\author{
YIHUA ZHANG ${ }^{1}$, ZHIDAN LUO $^{2}$, LIQUN MA $^{2}$, QIANG XU ${ }^{1}$, QIHONG YANG $^{1}$ and LIANGYI SI ${ }^{1}$ \\ ${ }^{1}$ Department of Geriatrics, Southwest Hospital, Third Military Medical University, Chongqing $400038 ;{ }^{2}$ Center for \\ Hypertension and Metabolic Diseases, Department of Hypertension and Endocrinology, Daping Hospital, \\ Third Military Medical University, Chongqing Institute of Hypertension, Chongqing 400042, P.R. China
}

Received December 29, 2009; Accepted March 3, 2010

DOI: $10.3892 /$ ijmm_00000398

\begin{abstract}
Advanced glycosylation end products (AGE) and its receptor (RAGE) axis is involved in the regulation of lipid homeostasis and is critical in the pathogenesis of diabetic atherosclerosis. We investigated the protective role of resveratrol against the AGE-induced impairment on macrophage lipid homeostasis. In THP-1-derived macrophages, RAGE was dose-dependently induced by AGE and played a key role in the AGE-induced cholesterol accumulation. Resveratrol markedly reduced RAGE expression via peroxisome proliferator-activated receptor (PPAR) $\gamma$ but not PPAR $\alpha$ or AMP-activated protein kinase. Importantly, pretreatment with resveratrol significantly ameliorated AGE-induced upregulation of scavenger receptor-A (SR-A) and down-regulation of ATP-binding cassette (ABC) A1 and ABCG1 and thus effectively prevented the cholesterol accumulation in macrophages as shown by cellular cholesterol analysis and oil red $\mathrm{O}$ staining. Moreover, blockade of PPAR $\gamma$ abolished all these effects of resveratrol. Collectively, our results indicate that resveratrol prevents the impairment of AGE on macrophage lipid homeostasis partially by suppressing RAGE via PPAR $\gamma$ activation, which might provide new insight into the protective role of resveratrol against diabetic atherosclerosis.
\end{abstract}

\section{Introduction}

Diabetes is associated with high frequency and high severity of atherosclerosis which causes most morbidity and mortality in diabetic patients $(1,2)$. This highlights the importance and

Correspondence to: Dr Liangyi Si, Department of Geriatrics, Southwest Hospital, Third Military Medical University, 29 Gaotanyan Main Street, Shapingba District, Chongqing 400038, P.R. China E-mail: liangyi_si@126.com

Key words: resveratrol, advanced glycosylation end products, lipid accumulation, peroxisome proliferator-activated receptor $\gamma$, macrophage, receptor for advanced glycosylation end products urgency of studying the mechanism of diabetic atherosclerosis and exploring therapeutic options.

Diabetic patients have increased production and reduced clearance of advanced glycosylation end products (AGE) (3). It has been well documented that AGE and its receptor (RAGE) axis is involved in the pathogenesis of cardiovascular disease due to oxidative stress and inflammatory responses (4-7). Furthermore, impaired macrophage lipid metabolism directly participates in foam cell formation in diabetes $(8,9)$. Recent studies suggest that AGE-RAGE interaction affects the expression of some scavenger receptors such as CD36, SR-A and lipid transporters like ATP-binding cassette (ABC)A1 and G1 (10-13). However, these studies were inconsistent and whether the macrophage lipid accumulation was actually affected by RAGE activation is still unclear.

Resveratrol (trans-3,5,4'-trihydroxystilbene, RESV), a natural polyphenol phytoalexin, possesses various bioactivities, including estrogenic, anti-platelet, anti-inflammatory and antioxidant effects (14). A recent study showed that resveratrol inhibited atherosclerosis in diabetic LDL deficient mice through lipid reduction via AMP activated kinase (AMPK) activation in hepatocytes (15). Another study indicated that resveratrol inhibits foam cell formation via suppression of reactive oxygen species (ROS) generation and macrophage activation (16). However, it is still unknown whether resveratrol prevents the impairment of AGE on the lipid homeostasis in macrophages, which might partially contribute to the prevention of diabetic atherosclerosis.

Resveratrol is able to selectively activate peroxisome proliferators-activated receptors (PPAR) (17-19). It has been shown that activation of PPAR $\gamma$ inhibited the expression of RAGE in mesangial cells, endothelial cells, hepatocytes and vascular smooth muscle cells (20-22). We hypothesized that resveratrol down-regulates RAGE expression and prevents the impairment of AGE on the macrophage lipid homeostasis through PPAR $\gamma$ activation. Therefore, we investigated the effects of AGE with or without pretreatment of resveratrol on the lipid accumulation in THP-1-derived macrophages treated with oxidized low-density lipoprotein (ox-LDL). 


\section{Materials and methods}

Materials. Phorbol 12-myristate 13-acetate (PMA), resveratrol, PPAR $\gamma$ inhibitor GW9662, PPAR $\alpha$ inhibitor GW6471 and AMPK inhibitor compound $\mathrm{C}$ were all purchased from Sigma-Aldrich Co. (USA). Antibodies against human CD36, SR-A, ABCA1 and ABCG1 from Santa Cruz (USA) were used for immunoblotting. Neutralizing antibody against human RAGE (R\&D Systems, USA) was used to block bioactivity of AGE and also used for immunoblotting. All other chemicals were commercially available.

Preparation of AGE. AGE was prepared as previously described (7). Bovine serum albumin (BSA) was dissolved with $0.01 \mathrm{~mol} / \mathrm{l}$ phosphate-buffered saline (PBS) (concentration of $5 \mathrm{~g} / \mathrm{l}$ ), incubated with $0.05 \mathrm{~mol} / \mathrm{l} \mathrm{D}$-glucose in $5 \% \mathrm{CO}_{2} / 95 \%$ air at $37^{\circ} \mathrm{C}$ for 12 weeks. Then unincorporated glucose was removed by dialysis overnight against PBS. AGE was identified by measuring AGE specific fluorescence at excitation/emission $=370 \mathrm{~nm} / 440 \mathrm{~nm}$ using a fluorescence spectrophotometer (Photon Technology International, USA). Fluorescence intensity of AGE was $>60$ times higher than that of BSA. AGE content was estimated by fluorescence intensity at a protein concentration of $1 \mathrm{~g} / \mathrm{l}$.

Cell culture and treatment. Human monocytic leukemia THP-1 cells obtained from American Type Culture Collection (ATCC) were cultured in RPMI-1640 (Gibco) supplemented with $10 \%$ fetal bovine serum (Gibco). THP-1 cells were stimulated with $0.1 \mu \mathrm{mol} / 1$ PMA for $24 \mathrm{~h}$ to induce monocyticmacrophage differentiation. Then macrophages were incubated with ox-LDL $(50 \mu \mathrm{g} / \mathrm{ml})$ in combination of BSA or AGE for $24 \mathrm{~h}$ to induce lipid accumulation. In treated conditions, macrophages were pretreated with resveratrol in the presence or absence of its inhibitors for $2 \mathrm{~h}$ before addition of ox-LDL and AGE.

Cellular cholesterol analysis. Cellular cholesterol and cholesterol ester (CE) were extracted and measured using a fluorometric assay (Cholesterol/CE Quantitation Kit, BioVision, USA), according to the manufacturer's instructions (23). Cells were counted with a counting plate before subjected to chloroform/methanol extraction to isolate cellular lipids. Fluorescence was measured at excitation/emission $=535 \mathrm{~nm} /$ $590 \mathrm{~nm}$ in a microplate reader. CE contents per $10^{6}$ cells were calculated.

Immunoblot analysis. Immunoblot analysis was performed as previously described (24). Cells were lysed in high-salt extraction buffer $(0.5 \mathrm{~mol} / \mathrm{l}$ Tris, $1 \%$ NP40, $1 \%$ TritionX-100, $1 \mathrm{~g} / \mathrm{l}$ sodium dodecyl sulfate, $1.5 \mathrm{~mol} / 1 \mathrm{NaCl}, 0.2 \mathrm{~mol} / \mathrm{l} \mathrm{EDTA}$, $0.01 \mathrm{~mol} / \mathrm{l} \mathrm{EGTA}$ ) plus $0.2 \mathrm{mmol} / 1$ protease inhibitor, placed at $-20^{\circ} \mathrm{C}$ for $20 \mathrm{~min}$, and centrifuged at $12,000 \mathrm{x}$ g at $4^{\circ} \mathrm{C}$ for $20 \mathrm{~min}$ to remove insoluble material. Protein concentrations were determined using a DC protein assay kit (Bio-Rad, USA). Lysates $(20 \mu \mathrm{g})$ were separated by SDS-PAGE, transferred to polyvinylidene difluoride membranes, and probed with the antibody against indicated protein. After incubation with secondary antibodies for $2 \mathrm{~h}$, proteins were detected by enhanced chemiluminescence and quantified using a Gel Doc 2000 Imager (Bio-Rad). $\beta$-actin was used as a loading
A
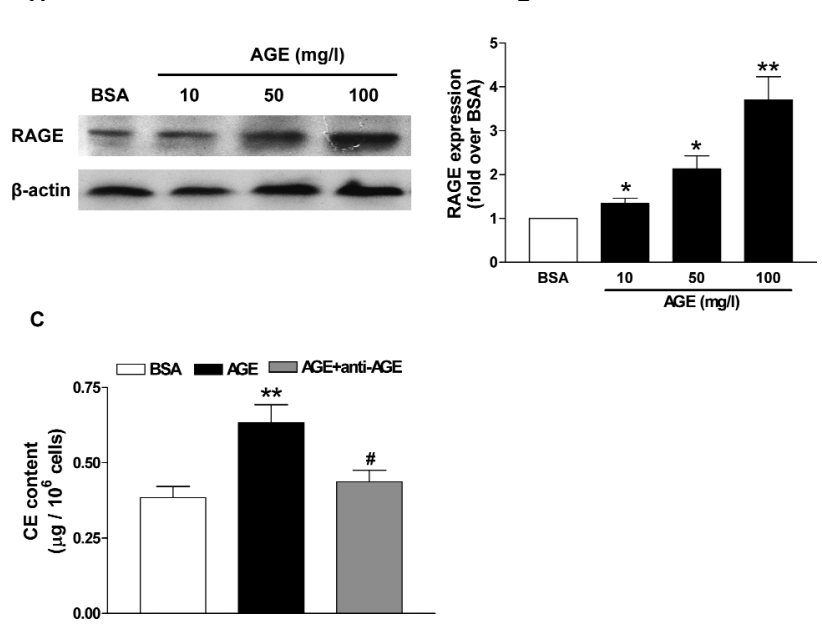

Figure 1. AGE promotes cholesterol accumulation through induction of RAGE in macrophages. (A) RAGE protein in macrophages incubated with indicated concentrations of AGE for $24 \mathrm{~h}$ was detected by immunoblotting. BSA (100 mg/l) was used as a negative control of AGE. (B) Summary data expressed as fold induction relative to BSA. ${ }^{*} \mathrm{P}<0.05,{ }^{* *} \mathrm{P}<0.01$ vs. BSA $(\mathrm{n}=3)$. (C) Cholesterol ester content per $10^{6}$ cells. Macrophages were incubated with ox-LDL for $24 \mathrm{~h}$ in the presence or absence of AGE or anti-AGE neutralizing antibody (anti-AGE, $50 \mathrm{mg} / \mathrm{l}) .{ }^{* *} \mathrm{P}<0.01$ vs. BSA, ${ }^{\#} \mathrm{P}<0.05$ vs. AGE $(\mathrm{n}=6)$.

control. Proteins of interest were expressed as the ratio against $\beta$-actin.

Oil-red O staining. Oil-red O staining of lipid droplets was performed as previously described (24). Cells were fixed with $4 \%$ formaldehyde in PBS and then stained for 30 min with $0.05 \%$ oil-red $\mathrm{O}$ in isopropanol and water (3:2 by volume). Cells were then washed with water and viewed by microscopy.

Statistics. All values are mean \pm SEM of at least 3 separated experiments. Comparisons between groups were analyzed using Student's t-test or one-way ANOVA with Bonferroni's multiple comparison post hoc tests as appropriate (SPSS Inc., USA). Two-sided p-values $<0.05$ were considered statistically significant.

\section{Results}

$A G E$ up-regulated RAGE expression and promoted cholesterol accumulation in macrophages. After $24 \mathrm{~h}$ incubation, AGE dose-dependently increased RAGE protein expression in macrophages (Fig. 1A and B), whereas the unmodified BSA had no effect on RAGE protein expression (data not shown). AGE had the most significant impact on the expression of RAGE at a concentration of $100 \mathrm{mg} / \mathrm{l}$ (Fig. 1A and B). In order to examine whether AGE affects cholesterol accumulation via RAGE, macrophages were treated with AGE in the presence or absence of anti-RAGE neutralizing antibody $(50 \mathrm{mg} / \mathrm{l})$ when incubated with ox-LDL for $24 \mathrm{~h}$. As shown in Fig. 1C, AGE $(100 \mathrm{mg} / \mathrm{l})$ significantly increased the cellular CE level. This increase was markedly blunted by the neutralizing anti-RAGE antibody, suggesting the key role of RAGE in the AGEinduced acceleration of cholesterol accumulation in macrophages. 

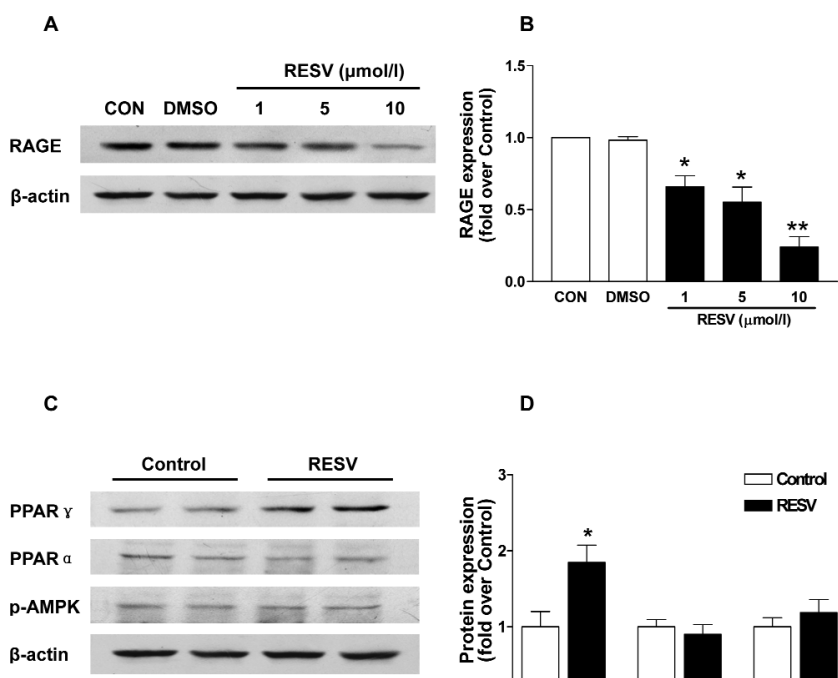

E

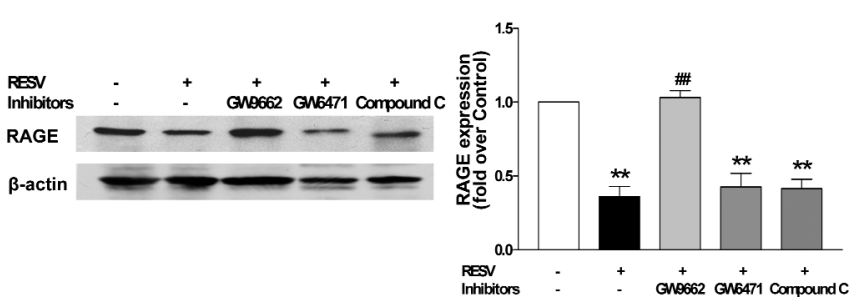

Figure 2. Resveratrol reduces AGE-induced RAGE expression via activation of PPAR $\gamma$ but not PPAR $\alpha$ or AMPK. (A) AGE-induced RAGE expression in macrophages pretreated with indicated concentrations of resveratrol (RESV). Macrophages without pretreatment $(\mathrm{CON})$ and pretreated with $10 \mu \mathrm{mol} / 1$ DMSO (DMSO) were both used as controls. Proteins were detected by immunoblotting. (B) Summary data of (A) expressed as fold induction over CON. ${ }^{*} \mathrm{P}<0.05,{ }^{* *} \mathrm{P}<0.01$ vs. CON (n=3). (C) Protein expression of PPAR $\gamma$, PPAR $\alpha$ and p-AMPK in macrophages without (control) or with resveratrol treatment (RESV) detected by immunoblotting. (D) Summary data of (C) shown as fold induction over control. ${ }^{*} \mathrm{P}<0.05$ vs. control $(\mathrm{n}=4)$. (E) AGEinduced RAGE expression in macrophages pretreated with resveratrol in the presence or absence of PPAR $\gamma$ inhibitor GW9662 (1 $\mu \mathrm{mol} / 1)$, PPAR $\alpha$ inhibitor GW6471 (10 $\mu \mathrm{mol} / \mathrm{l})$ or AMPK inhibitor compound C $(1 \mu \mathrm{mol} / \mathrm{l})$. Proteins were detected by immunoblotting. (F) Summary data of (E) expressed as fold relative to control group. ${ }^{* *} \mathrm{P}<0.01$ vs. control group without pretreatment with resveratrol or inhibitors. ${ }^{\# \#} \mathrm{P}<0.01$ vs. control group with resveratrol pretreatment $(n=3)$.

Resveratrol reduces RAGE expression via activation of PPAR but not PPARa or AMPK. As shown in Fig. 2A and B, resveratrol pretreatment led to a dose-dependent suppression of AGE-induced RAGE expression. Resveratrol $(10 \mu \mathrm{mol} / \mathrm{l})$ suppressed the expression of RAGE by $\sim 75 \%$, whereas dimethyl sulfoxide (DMSO) had little effect on RAGE expression. Then we detected the expression of PPAR $\gamma$, PPAR $\alpha$ and phosphorylated AMPK (p-AMPK), the potential targets of resveratrol. We found that only PPAR $\gamma$ expression was up-regulated by resveratrol, PPAR $\alpha$ and p-AMPK expression remained unchanged (Fig. $2 \mathrm{C}$ and D). The key role of PPAR $\gamma$ was further confirmed by using selective inhibitors. As shown in Fig. 2E and F, the effect of resveratrol on RAGE expression was significantly blunted by the presence of GW9662, a selective antagonist of PPAR $\gamma$. However, the antagonists of PPAR $\alpha$ and AMPK, GW6471 and compound C, failed to inhibit the effect of resveratrol. These results suggest
A

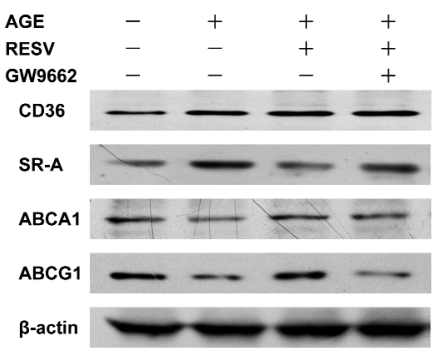

B

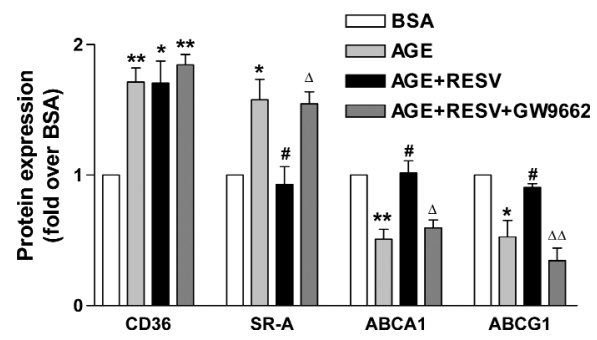

Figure 3. Resveratrol reverses the effect of AGE on the expression of proteins involved in lipid homeostasis via PPAR $\gamma$ activation. (A) Representative figures showing the protein expression of CD36, SR-A, ABCA1 and ABCG1 in the macrophages treated with or without AGE in the presence or absence of resveratrol and GW9662. Proteins were detected by immunoblotting. (B) Summary data of at least 3 independent experiments. ${ }^{*} \mathrm{P}<0.05,{ }^{* *} \mathrm{P}<0.01$ vs. BSA; ${ }^{*} \mathrm{P}<0.05$ vs. AGE; $\triangle \mathrm{P}<0.05, \triangle \triangle \mathrm{P}<0.01$ vs. AGE+RESV.

that the suppression of resveratrol on macrophage RAGE expression was PPAR $\gamma$-dependent.

Resveratrol reverses the effect of AGE on the expression of proteins involved in lipid homeostasis via PPAR activation. The balance of cholesterol uptake and reverse cholesterol transport could have been broken due to the AGE-induced molecular modulation. As shown in Fig. 3A and B, incubation of macrophages with AGE resulted in significant up-regulation of CD36 and SR-A and down-regulation of ABCA1 and ABCG1. As expected, pretreatment with resveratrol significantly ameliorated the AGE-induced up-regulation of SR-A and down-regulation of ABCA1 and ABCG1 (Fig. 3A and $\mathrm{B})$. Moreover, these effects of resveratrol were abolished by PPAR $\gamma$ antagonist. However, resveratrol had little effect on AGE-induced CD36 expression. These results indicate that resveratrol partially reversed the effects of AGE on the proteins involved in lipid uptake and transport.

Resveratrol prevents the AGE-induced acceleration of cholesterol accumulation and inhibits foam cell formation via PPAR . As CD36- and SR-A-mediated cholesterol influx was enhanced and ABCA1- and ABCG1-mediated cholesterol efflux was reduced, cholesterol accumulation was significantly aggravated by AGE incubation (Fig. 4A). However, pretreatment with resveratrol reversed the AGE-induced changes in ABCA1, ABCG1 and SR-A and thus effectively prevented cholesterol accumulation in macrophages (Fig. 4A). Additionally, the resveratrol-induced reduction in cellular CE was blunted by PPAR $\gamma$ antagonist (Fig. 4A).

Finally, we examined the effects of resveratrol and AGE on macrophage-derived foam cell formation using oil-red $\mathrm{O}$ 
A

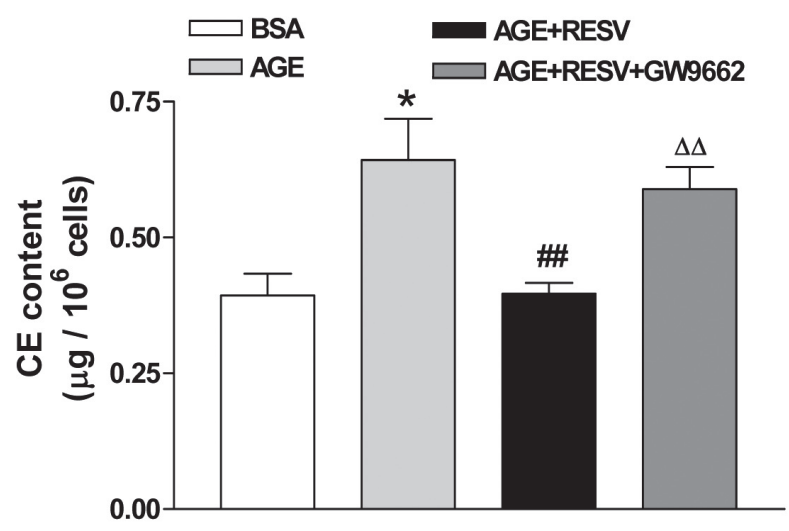

B

BSA

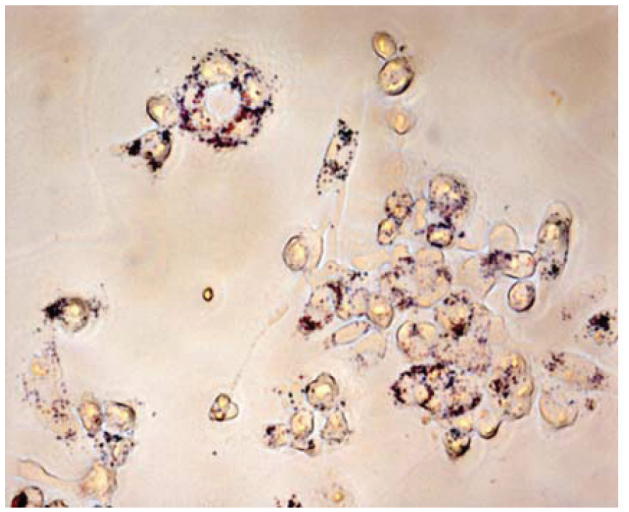

AGE+RESV

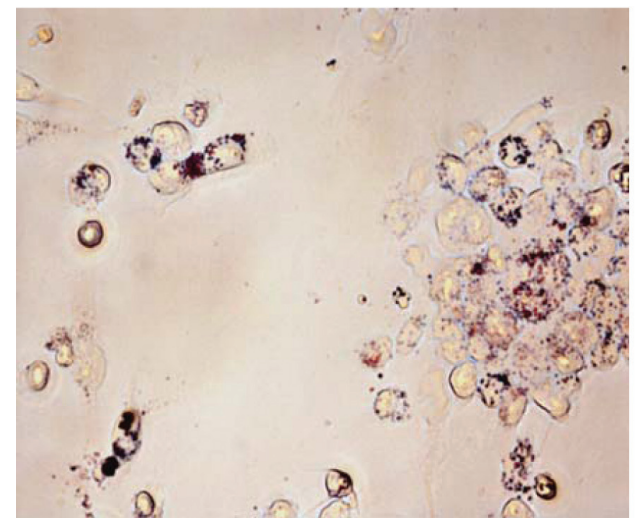

AGE

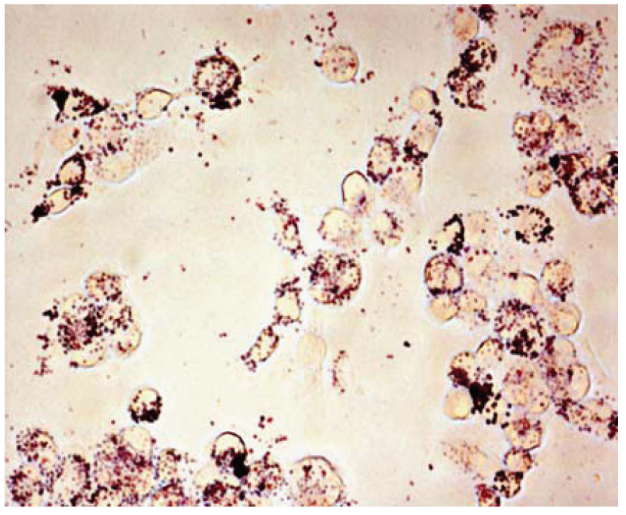

AGE+RESV+GW9662

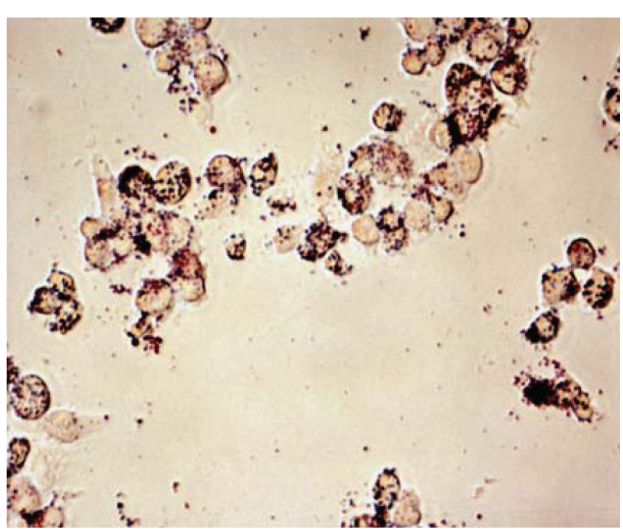

Figure 4. Resveratrol prevents the AGE-induced acceleration of cholesterol accumulation and inhibits foam cell formation via PPAR $\gamma$. (A) Cholesterol ester content per $10^{6}$ cells. Macrophages were incubated with ox-LDL in the presence or absence of AGE, RESV and GW9662. ${ }^{*} \mathrm{P}<0.05$ vs. BSA, ${ }^{\# \#} \mathrm{P}<0.01$ vs. AGE, $\triangle \triangle \mathrm{P}<0.01$ vs. AGE+RESV ( $\mathrm{n}=6$ ). (B) Representative pictures (magnification, $\mathrm{x} 400$ ) showing the lipid accumulation in macrophages incubated with ox-LDL in the presence or absence of AGE, RESV and GW9662.Lipid droplets were stained in red by oil-red O. Experiments were performed in triplicate for each group.

staining. As shown in Fig. 4B, incubation of macrophages with ox-LDL alone for $24 \mathrm{~h}$ elicited slight lipid accumulation in macrophages, whereas incubation together with AGE produced a great deal of massive lipid droplets in cells, resulting in typical formation of foam cells (Fig. 4B). As expected, resveratrol (10 $\mu \mathrm{mol} / \mathrm{l})$ pretreatment ameliorated the AGE-induced foam cell formation, which was blunted by the presence of PPAR $\gamma$ antagonist (Fig. 4B).

\section{Discussion}

Numerous studies have suggested the potential of resveratrol in prevention of diabetic cardiovascular diseases (14-16). However, the knowledge on the underlying mechanisms remains incomplete. AGE is reported to play a causative role in diabetic atherosclerosis mainly through oxidative stress and inflammatory response (4-6). The present study revealed that 
besides the pro-inflammatory effect, AGE aggravated macrophage lipid accumulation at least in part by regulating the expression of CD36, SR-A, ABCA1 and ABCG1 via interaction with RAGE. More importantly, this study, for the first time, showed that resveratrol can suppress RAGE expression via PPAR $\gamma$ activation, directly regulate lipid transporters, and thus prevent the impairment of AGE on lipid homeostasis in macrophages, which might suggest new approaches to prevent diabetic atherosclerosis.

RAGE plays a key role in the pathogenesis of AGE-related foam cell formation. It is well understood that the cholesterol uptake facilitated by scavenger receptors like SR-A and CD-36 and the cholesterol efflux mediated by ABCA1 and ABCG1 both directly contribute to the macrophage transformation into a foam cell $(8,9)$. Previous studies indicated that AGE increased the gene expression of two major ox-LDL receptors, SR-A and CD36 (10). RNA interference against RAGE led to a significant inhibition of CD36 expression in human monocytes (11). Isoda et al revealed that AGE reduced gene expression of ABCG1 but not ABCA1 in human macrophages, and an anti-RAGE antibody significantly limited ABCG1 reduction (13). However, none of the above studies observed the effects of AGE on both lipid influx and efflux and on the net lipid accumulation in macrophages. Our study confirmed that AGE dose-dependently induces expression of RAGE, increases expression of CD36 and SR-A, and simultaneously reduces expression of both $\mathrm{ABCA} 1$ and $\mathrm{ABCG} 1$. As a result of the above molecular modulation, AGE increased intracellular cholesterol content and the anti-RAGE antibody significantly limited the increase. This result was supported in part by another study in uremic mice showing that intraperitoneal injection of the anti-RAGE antibody reduced the aortic plaque area without affecting blood cholesterol and pressure (5). Our results implicate that AGE-RAGE aggravates macrophage lipid accumulation through molecular modulation of both cholesterol influx and efflux.

Resveratrol protects cardiovascular system from oxidative damage including the damage of AGE. Resveratrol is reported to reverse the AGE-induced proliferation of vascular smooth muscle cells and DNA synthesis $(25,26)$. However, whether the key pathogenic factor in diabetes, RAGE, is regulated by resveratrol is still unclear. Recently, several studies suggested that PPAR $\gamma$ inhibited RAGE expression in several other types of cells (20-22). Ma et al reported that grape seed proanthocyanidin extracts, an analog of resveratrol, up-regulated PPAR $\gamma$ and down-regulated RAGE in endothelial cells (27), but the fundamental role of PPAR $\gamma$ needed further elucidation. Thus we postulated that resveratrol might suppress macrophage RAGE expression through PPAR $\gamma$ activation. As expected, we found that resveratrol reduced macrophage RAGE expression and increased PPAR $\gamma$ expression. In addition, we elucidated that PPAR $\gamma$ was necessary in the effect of resveratrol on macrophage RAGE expression. Using specific antagonists, we further excluded the participation of PPAR $\alpha$ and AMPK, the other two possible targets of resveratrol related to lipid metabolism $(15,28)$.

More importantly, we found that resveratrol effectively prevented the adverse effect of AGE on the expression of SR-A, ABCA1 and ABCG1 through PPAR $\gamma$ activation. However, the increase of CD36 expression elicited by AGE was not attenuated by resveratrol. The distinct effect of resveratrol on CD36 expression might be attributed to the divergent effects of PPAR $\gamma$ on different scavenger receptors. Moore et al indicated that PPAR $\gamma$ activation up-regulated CD36 expression but leads to compensatory down-regulation of SR-A, which results in unchanged lipid content in macrophages (29). Although the role of PPAR $\gamma$, independent of RAGE, in foam cell formation is still controversial, recent studies tend to support that PPAR $\gamma$ activation inhibits or at least does not promote foam cell formation (28-30). Thus, it is possible that resveratrol might exert protective effects not only through the modulation of RAGE but also through the direct actions of PPAR $\gamma$. As a result of the molecular modulation involved in lipid uptake and efflux, the aggravated lipid accumulation induced by AGE was prevented by resveratrol through PPAR $\gamma$ activation. Our hypothesis was finally confirmed by cholesterol analysis and oil-red $\mathrm{O}$ staining of macrophagederived foam cells.

In conclusion, our results strongly indicate that resveratrol prevents the AGE-induced acceleration of macrophage lipid accumulation through suppression of RAGE via PPAR $\gamma$ activation. Our study for the first time revealed the effects of resveratrol on RAGE expression and lipid homeostasis in macrophages, which are critical for the foam cell formation in diabetes. These findings might be a novel mechanism in part to explain the protective role of resveratrol and provide new evidence for the application of resveratrol in the prevention of diabetic atherosclerosis.

\section{Acknowledgements}

This work was supported by the Chongqing Natural Science Foundation in China (No. CSTC 2007BB5384).

\section{References}

1. Beckman JA, Creager MA and Libby P: Diabetes and atherosclerosis: epidemiology, pathophysiology, and management. JAMA 287: 2570-2581, 2002.

2. King H, Aubert RE and Herman WH: Global burden of diabetes, 1995-2025: prevalence, numerical estimates, and projections. Diabetes Care 21: 1414-1431, 1998.

3. Wu JT: Advanced glycosylation end products: a new disease marker for diabetes and aging. J Clin Lab Anal 7: 252-255, 1993.

4. Yamagishi S, Nakamura K, Matsui T, Noda Y and Imaizumi T: Receptor for advanced glycation end products (RAGE): a novel therapeutic target for diabetic vascular complication. Curr Pharm Des 14: 487-495, 2008

5. Bro S, Flyvbjerg A, Binder CJ, et al: A neutralizing antibody against receptor for advanced glycation end products (RAGE) reduces atherosclerosis in uremic mice. Atherosclerosis 201: 274-280, 2008.

6. Csiszar A and Ungvari Z: Endothelial dysfunction and vascular inflammation in type 2 diabetes: interaction of AGE/RAGE and TNF- $\alpha$ signaling. Am J Physiol Heart Circ Physiol 295: H475-H476, 2008.

7. Ge J, Jia Q, Liang C, et al: Advanced glycosylation end products might promote atherosclerosis through inducing the immune maturation of dendritic cells. Arterioscler Thromb Vasc Biol 25: 2157-2163, 2005.

8. Cuchel M and Rader DJ: Macrophage reverse cholesterol transport: key to the regression of atherosclerosis? Circulation 113: 2548-2555, 2006.

9. Shashkin P, Dragulev B and Ley K: Macrophage differentiation to foam cells. Curr Pharm Des 11: 3061-3072, 2005.

10. Iwashima Y, Eto M, Hata A, et al: Advanced glycation end products-induced gene expression of scavenger receptors in cultured human monocyte-derived macrophages. Biochem Biophys Res Commun 277: 368-380, 2000. 
11. Xanthis A, Hatzitolios A, Fidani S, Befani C, Giannakoulas G and Koliakos G: Receptor of advanced glycation end products (RAGE) positively regulates CD36 expression and reactive oxygen species production in human monocytes in diabetes. Angiology 60: 772-779, 2009.

12. Passarelli M, Tang C, McDonald TO, et al: Advanced glycation end product precursors impair ABCA1-dependent cholesterol removal from cells. Diabetes 54: 2198-2205, 2005.

13. Isoda K, Folco EJ, Shimizu K and Libby P: AGE-BSA decreases ABCG1 expression and reduces macrophage cholesterol efflux to HDL. Atherosclerosis 192: 298-304, 2007.

14. Sadruddin S and Arora R: Resveratrol: biologic and therapeutic implications. J Cardiometab Syndr 4: 102-106, 2009.

15. Zang M, Xu S, Maitland-Toolan KA, et al: Polyphenols stimulate AMP-activated protein kinase, lower lipids, and inhibit accelerated atherosclerosis in diabetic LDL receptordeficient mice. Diabetes 55: 2180-2191, 2006.

16. Park DW, Baek K, Kim JR, et al: Resveratrol inhibits foam cell formation via NADPH oxidase 1-mediated reactive oxygen species and monocyte chemotactic protein-1. Exp Mol Med 41: 171-179, 2009

17. Ulrich S, Loitsch SM, Rau O, et al: Peroxisome proliferatoractivated receptor $\gamma$ as a molecular target of resveratrol-induced modulation of polyamine metabolism. Cancer Res 66: 7348-7354, 2006.

18. Cheng G, Zhang X, Gao D, Jiang X and Dong W: Resveratrol inhibits MMP-9 expression by up-regulating PPAR $\gamma$ expression in an oxygen glucose deprivation-exposed neuron model. Neurosci Lett 451: 105-108, 2009.

19. Kennedy A, Overman A, Lapoint K, et al: Conjugated linoleic acid-mediated inflammation and insulin resistance in human adipocytes are attenuated by resveratrol. J Lipid Res 50: 225-232, 2009.

20. Wang K, Zhou Z, Zhang M, et al: Peroxisome proliferatoractivated receptor $\gamma$ down-regulates receptor for advanced glycation end products and inhibits smooth muscle cell proliferation in a diabetic and nondiabetic rat carotid artery injury model. J Pharmacol Exp Ther 317: 37-43, 2006.

21. Matsui T, Yamagishi S, Takeuchi M, Ueda S, Fukami K and Okuda S: Nifedipine, a calcium channel blocker, inhibits advanced glycation end product (AGE)-elicited mesangial cell damage by suppressing AGE receptor (RAGE) expression via peroxisome proliferator-activated receptor- $\gamma$ activation. Biochem Biophys Res Commun 385: 269-272, 2009.
22. Yoshida T, Yamagishi S, Nakamura K, et al: Telmisartan inhibits AGE-induced C-reactive protein production through downregulation of the receptor for AGE via peroxisome proliferatoractivated receptor- $\gamma$ activation. Diabetologia 49: 3094-3099, 2006.

23. Ouimet M, Wang MD, Cadotte N, Ho K and Marcel YL: Epoxycholesterol impairs cholesteryl ester hydrolysis in macrophage foam cells, resulting in decreased cholesterol efflux. Arterioscler Thromb Vasc Biol 28: 1144-1150, 2008.

24. Zhang LL, Yan Liu D, Ma LQ, et al: Activation of transient receptor potential vanilloid type-1 channel prevents adipogenesis and obesity. Circ Res 100: 1063-1070, 2007.

25. Mizutani K, Ikeda K, Nishikata T and Yamori Y: Phytoestrogens attenuate oxidative DNA damage in vascular smooth muscle cells from stroke-prone spontaneously hypertensive rats. J Hypertens 18: 1833-1840, 2000.

26. Mizutani K, Ikeda K and Yamori Y: Resveratrol inhibits AGEsinduced proliferation and collagen synthesis activity in vascular smooth muscle cells from stroke-prone spontaneously hypertensive rats. Biochem Biophys Res Commun 274: 61-67, 2000.

27. Ma L, Gao HQ, Li BY, Ma YB, You BA and Zhang FL: Grape seed proanthocyanidin extracts inhibit vascular cell adhesion molecule expression induced by advanced glycation end products through activation of peroxisome proliferators-activated receptor $\gamma$. J Cardiovasc Pharmacol 49: 293-298, 2007.

28. Li AC, Binder CJ, Gutierrez A, et al: Differential inhibition of macrophage foam-cell formation and atherosclerosis in mice by PPAR $\alpha$, beta/delta, and $\gamma$. J Clin Invest 114: 1564-1576, 2004.

29. Moore KJ, Rosen ED, Fitzgerald ML, et al: The role of PPARin macrophage differentiation and cholesterol uptake. Nat Med 7: 41-47, 2001

30. Tontonoz P, Nagy L, Alvarez JG, Thomazy VA and Evans RM: PPAR $\gamma$ promotes monocyte/macrophage differentiation and uptake of oxidized LDL. Cell 93: 241-252, 1998. 